\title{
The Development of a Model for Decision Support System of Assessment and Selection of University Academic Staff
}

\author{
Irakli Basheleishvili*, Avtandil Bardavelidze ${ }^{1}$, Sergo Tsiramua² \\ ${ }^{*}$ Akaki Tsereteli State University, Kutaisi, Georgia \\ ${ }^{2}$ University of Georgia, Tbilisi, Georgia
}

\begin{abstract}
The paper deals with the development of the assessment, ranking and selection model of the university's academic staff. To develop this model, there was used the multi-criterion analysis method TOPSIS, which is based on the theory of fuzzy sets. Based on this model, we can establish the decision-making support system for the assessment and selection of academic staff on the basis of the expert assessments
\end{abstract}

Keywords: Academic staff, fuzzy number, TOPSIS, decision matrix, assessment, selection.

\section{Introduction}

The university's academic staff is one of the most important links carrying out research and educational activities. Academic staff consists of professors. The professors are composed of full professor, associate professor and assistant professor. The quality of scientific and educational activities at the university is directly linked to the qualification of academic staff. That is why the universities seek to staff particular teaching areas adequately. The assessment of the qualification of academic staff is done mainly when the university announces the competition for the vacancies of academic positions by means provided for in the legislation (Moses, 1988).

The academic positions can only be taken through an open competition, which should comply with the principles of transparency, equity and fair competition. Holding the competitions for the vacancies of academic positions based on the principles of transparency, equity and fair competition is ensured by a temporary collegiate body - the Competition Commission (a group composed of qualified experts). The Competition Commission will review and assess the documents submitted by the applicants to confirm their suitability for the academic position.

It is important for the Competition Commission to carry out its activities efficiently and objectively, in order to avoid the mistakes in the academic staff selection process.

To ensure the objective and successful academic staff selection process, we consider it advisable to establish the decision-making support system, through which the Competition Commission will carry out its activities.

The decision-making support system that provides the academic staff assessment and selection process needs a model, through which the system will provide the assessment and selection of academic staff for a particular area or position, on the basis of the assessment of experts (the Competition Commission members). The aim of our work is to elaborate a model of the assessment and selection, on the basis of which we will be able to design the decision-making support system for the assessment and selection of academic staff.

There are numerous scientific papers with insight into the issues of the assessment and selection of human resources, which is not the case in the assessment and selection of the University's academic staff. stands to 
reason that, the academic staff is a human resource, but the academic staff differs greatly from regular personnel. Consequently, the assessment and selection of academic staff process is a specific process and differs radically different from the regular personnel assessment and selection process (Basheleishvili, \& Bardavelidze, 2018; Meinert, Davis, 1989; Keenan, McGarraghy, McNamara, Phelan, \& Schools,2004 ).

\section{Basic Part}

The objective of the assessment and selection of academic staff is similar to the task of multicriteria decision, which is based on a decision matrix, which in turn consists of the alternatives and criteria. the alternatives are a choice from a broad range of options that should be assessed for the selection of the best option. The criteria are the characteristics of the alternatives, which are used to assess the alternatives. (Basheleishvili, 2018; Basheleishvili, \& Bardavelidze, 2018).

In this case, we should draw up a decision matrix according to each specialty and academic position, in which the alternatives will be the applicants (for the academic positions), while the criteria are the assessment criteria. The values of the assessment criteria are determined by the experts who are involved in the Competition Commission.

With regard to the assessment and selection, we use the Fuzzy - TOPSIS expert method. The Fuzzy-TOPSIS expert method is one of the effective tools that helps the decision-makers and experts in the formulation of their goals and subjective considerations, as well as in using fuzzy mathematics, linguistic variables and the phasesets in the decision-making process for the assessment of the alternatives and criteria. (Sun, Lin , 2009; Madi, Tap, \& Osman, 2011).

We can formulate the objectives of the assessment and selection of academic staff in the following way: we have the $L=\left\{L_{1}, L_{2}, \ldots L_{g}\right\}$ set of specialties, for which we are going to provide the assessment and selection of academic staff; $C^{\prime}{ }_{i 1}, C^{\prime}{ }_{i 2}, \ldots C^{\prime}{ }_{i n}$ - criteria for the assessment of full professor for $i$-specialty; $C^{\prime \prime}{ }_{i 1}, C^{\prime \prime}{ }_{i 2}, \ldots C^{\prime \prime}{ }_{i n}$ - criteria for the assessment of associate professor for $i$-specialty; $C^{\prime \prime \prime}{ }_{i 1}, C^{\prime \prime \prime}{ }_{i 2}, \ldots C^{\prime \prime \prime}{ }_{i n}$ - criteria for the assessment of assistant professor for $i$ specialty; $W^{\prime}{ }_{i 1}, W^{\prime}{ }_{i 2}, \ldots W^{\prime}{ }_{i n}$ - the criterion weights for the assessment of full professor for $i$-specialty; $W^{\prime \prime}{ }_{i 1}, W^{\prime \prime}{ }_{i 2}, \ldots W^{\prime \prime}{ }_{i n}$ - the criterion weights for the assessment of associate professor for $i$-specialty; $W^{\prime \prime \prime}{ }_{i 1}, W^{\prime \prime \prime}{ }_{i 2}, \ldots W^{\prime \prime \prime}{ }_{i n}$ - the criterion weights for the assessment of assistant professor for $i$-specialty.

$E_{i}=\left\{e_{i 1}, e_{i 2}, \ldots e_{i t}\right\}$ - a set of experts by specialties; $P_{i}^{\prime}=\left\{p_{i 1}^{\prime}, p_{i 2}^{\prime}, \ldots p_{i m}^{\prime}\right\}$ - a set of applicants for the academic position of full professor for $i$-specialty; $P_{i}^{\prime \prime}=\left\{p_{i 1}^{\prime \prime}, p_{i 2}^{\prime \prime}, \ldots p_{i m}^{\prime \prime}\right\} \quad$ - a set of applicants for the academic position of associate professor for $i$-specialty; $P_{i}^{\prime \prime \prime}=\left\{p_{i 1}^{\prime \prime \prime}, p_{i 2}^{\prime \prime \prime}, \ldots p_{i m}^{\prime \prime \prime}\right\} \quad$ - set of applicants for the academic position of assistant professor. $N_{i}^{\prime}$ - number of professors to be selected for $i$-specialty; $N_{i}^{\prime \prime}$ - number of associate professors to be selected for $i$-specialty; $N_{i}^{\prime \prime \prime}$ - number of assistant professors to be selected for $i$-specialty.

Thus, our goal is to draw up a decision matrix for each specialty and academic position, based on the defined assessment criteria, their alternatives and fuzzy expert assessments(triangular fuzzy number ( Zhang, Ma, \& Chen, 2014)), as well as to implement each of them for the selection of academic staff.

A triangular fuzzy number is represented as a triplet $\tilde{a}=(a, b, c)$. The membership function $\mu_{\tilde{a}}(x)$ of triangular fuzzy number $\tilde{a}$ is given as :

$$
\mu_{\tilde{a}}(x)=\left\{\begin{array}{c}
\frac{x-a}{b-a} \quad \text { if } a \leq x \leq b \\
\frac{x-c}{b-c} \text { if } b \leq x \leq c \\
0 \text { otherwise }
\end{array}\right.
$$

For the expert assessments by fuzzy numbers, we use fuzzy numbers and the following scales of the appropriate linguistic variables (Table 1-2) (Madi, Tap, \& Osman, 2011).

Table 1. Linguistic variables for the importance weight of each criterion

\begin{tabular}{|c|c|}
\hline Linguistic variable & $\begin{array}{l}\text { Triangular } \\
\text { number }\end{array}$ \\
\hline Very Not Important (VNI) & $(0,0,0.1)$ \\
\hline Not Important (NI) & $(0,0.1,0.3)$ \\
\hline $\begin{array}{l}\text { Somewhat Not Important } \\
\text { (SNI) }\end{array}$ & $(0.1,0.3,0.5)$ \\
\hline Medium (M) & $(0.3,0.5,0.7)$ \\
\hline Somewhat Important (SI) & $(0.5,0.7,0.9)$ \\
\hline Important (I) & $(0.7 .0 .9,1.0)$ \\
\hline Very Important (VI) & $(0.9,1.0,1.0)$ \\
\hline
\end{tabular}

Table 2. Linguistic variables for the ratings

\begin{tabular}{lc}
\hline \multicolumn{1}{c}{ Linguistic variable } & Triangular fuzzy number \\
\hline Very Not Poor (VNP) & $(0,0,1)$ \\
Poor (P) & $(0,1.3)$ \\
Medium Poor (MP) & $(1,3,5)$ \\
Fair (F) & $(3,5,7)$ \\
Medium Good (MG)) & $(5,7,9)$ \\
Good (G) & $(7.9,10)$ \\
Very Good (VG) & $(9,10,0)$ \\
\hline
\end{tabular}

\section{The academic staff assessment and selection stages based on Fuzzy-Topsis:}

Stage 1. Let us draw up a decison matrix for a particular academic position for i-specialty, which is as follows: 
Basheleishvili et al. / The Development of a Model for Decision Support System of Assessment and Selection of University Academic Staff

$$
\begin{gathered}
D_{i}=\begin{array}{cccc}
C_{i 1} & C_{i 1} & \cdots & C_{i n} \\
p_{i 1} \\
\vdots \\
p_{i m}
\end{array}\left[\begin{array}{cccc}
\tilde{x}_{11} & \tilde{x}_{12} & \cdots & \tilde{x}_{1 n} \\
\tilde{x}_{21} & \tilde{x}_{22} & \cdots & \tilde{x}_{2 n} \\
\vdots & \vdots & \ddots & \vdots \\
\tilde{x}_{1 m} & \tilde{x}_{2 m} & \cdots & \tilde{x}_{n m}
\end{array}\right] \quad \mathrm{i}=1, \mathrm{~g} \\
\text { Where }
\end{gathered}
$$$$
\begin{array}{r}
\tilde{x}_{u v}=\frac{1}{s}\left(\tilde{x}_{u v}^{1}+\tilde{x}_{u v}^{2}+\cdots+\tilde{x}_{u v}^{s}\right) \\
\mathrm{u}=1, \mathrm{~m} . \quad \mathrm{v}=1, \mathrm{n} .
\end{array}
$$

Where $\tilde{x}_{u v}^{s}$ is the rating of alternative $p_{i u}$ with respect to criterion $c_{i v}$ evaluated by expert, and $\tilde{x}_{u v}^{s}=\left(a_{u v}^{s}, b_{u v}^{s}, c_{u v}^{s}\right)$.

Stage 2. Let us determine triangular fuzzy numbers of the criterion weights for a given specialty and academic position, which are assessed by the experts in accordance with Table 1.

Stage 3. Normalize the fuzzy decision matrix, the normalized fuzzy decision matrix denoted by $\widetilde{R_{\iota}}$ is shown as following formula:

$$
\begin{aligned}
\widetilde{R}_{l} & =\left[\tilde{r}_{k l}\right]_{m \times n} \\
\mathrm{k} & =1, \mathrm{~m} . \quad \mathrm{l}=1, \mathrm{n} .
\end{aligned}
$$

Then the normalization process can be performed by following formula:

Where

$$
\tilde{r}_{k l}=\left(\frac{a_{k l}}{c_{l}^{*}}, \frac{b_{k l}}{c_{l}^{*}}, \frac{c_{k l}}{c_{l}^{*}}\right)
$$

$$
c_{l}^{*}=\max _{k} c_{k l}
$$

The normalized $\tilde{r}_{k l}$ are still triangular fuzzy numbers.

Stage 4. The weighted fuzzy normalized decision matrix is shown as following matrix $\widetilde{V}_{l}$ :

$$
\begin{gathered}
\widetilde{V}_{l}=\left[\tilde{v}_{k l}\right]_{m \times n} \\
\tilde{v}_{k l}=\tilde{r}_{k l} \oplus \widetilde{w_{l}}
\end{gathered}
$$

Stage 5. Determine the fuzzy positive-ideal solution (FPIS) and fuzzy negative-ideal solution (FNIS) According to the weighted normalized fuzzy decision matrix, we know that the elements $\tilde{v}_{k l}$ are normalized positive TFNs and their ranges belong to the closed interval $[0,1]$. Then, we can define the FPIS $\mathrm{A}^{+}$and FNIS $A^{-}$as following formula (Sun, Lin, 2009):

$$
\begin{gathered}
A^{+}=\left(\tilde{v}_{1}^{+}, \tilde{v}_{2}^{+}, \ldots, \tilde{v}_{n}^{+}\right) \\
A^{-}=\left(\tilde{v}_{1}^{-}, \tilde{v}_{2}^{-}, \ldots, \tilde{v}_{n}^{-}\right)
\end{gathered}
$$

where ${\widetilde{v_{l}}}^{+}=(1,1,1)$ and ${\widetilde{v_{l}}}^{-}=(0,0,0)$.

Stage 6: Calculate the distance of each alternative from FPIS and FNIS the distances $\left(d_{k}^{+}\right.$and $\left.d_{k}^{-}\right)$of each alternative $A^{+}$from and $A^{-}$can be currently calculated by the area compensation method(Sun, Lin, 2009):

$$
\begin{gathered}
d_{k}^{+}=\sum_{l=1}^{n} d\left(\tilde{v}_{k l}, \widetilde{v}_{l}^{+}\right) \\
d_{k}^{-}=\sum_{l=1}^{n} d\left(\tilde{v}_{k l}, \widetilde{v}_{l}^{-}\right)
\end{gathered}
$$

Where $d(\tilde{a}, \tilde{b})$ denotes the Euclidean distance between two fuzzy numbers $\tilde{a}$ and $\tilde{b}$. (Szmidt, Eulalia, \& Kacprzyk, 2000).
Stage 7. The closeness coefficient $\boldsymbol{C}_{\boldsymbol{i}}$ is then calculated to determine the ranking of each alternative. The closeness coefficient is given by:

$$
\boldsymbol{C} \boldsymbol{C}_{\boldsymbol{i k}}=\frac{d_{k}^{-}}{d_{k}^{+}+d_{k}^{-}}
$$

Stage 8. Let us select $N_{i}$ number of applicants having the highest $\boldsymbol{C} \boldsymbol{C}_{\boldsymbol{i} \boldsymbol{k}}$ values for a particular position for $i$-specialty.

\section{Numerical experiment to demonstrate the work of the method presented in the paper}

Consider the case of the assessment and selection of academic staff, when we want to select three professors $\left(N_{1}^{\prime}=3\right)$ for the specialty of Software Engineering $\left(d_{1}\right.$ ), whose assessment criteria are as follows: suitability of the academic degree and scientific work with the vacancy profile $\left(C^{\prime}{ }_{11}\right)$; suitability of work and pedagogical experience with the vacancy profile $\left(C^{\prime}{ }_{12}\right)$; scientific research and research grant projects $\left(C^{\prime}{ }_{13}\right)$; portfolio $\left(C^{\prime}{ }_{14}\right)$; an interview with a Competition Commission $\left(C^{\prime}{ }_{15}\right)$; the Competition Commission for $d_{1}$ specialty is composed of three experts $e_{11}, e_{12}, e_{13}$.

The experts use the linguistic weighting variables (See Table 1.) for determining the level of importance of criteria (Table 3):

Table 3. Linguistic variables of the importance weight of the criteria

\begin{tabular}{cccc}
\hline & $e_{11}$ & $e_{12}$ & $e_{13}$ \\
\hline$C_{11}^{\prime}$ & $\mathrm{VI}$ & $\mathrm{VI}$ & $\mathrm{I}$ \\
$C_{12}^{\prime}$ & $\mathrm{SI}$ & $\mathrm{SI}$ & $\mathrm{I}$ \\
$C_{13}^{\prime}$ & $\mathrm{M}$ & $\mathrm{M}$ & $\mathrm{SNI}$ \\
$C_{14}^{\prime}$ & $\mathrm{SI}$ & $\mathrm{I}$ & $\mathrm{SI}$ \\
$C_{15}^{\prime}$ & $\mathrm{M}$ & $\mathrm{I}$ & $\mathrm{SNI}$ \\
\hline
\end{tabular}

Converting linguistic variables to the triangular fuzzy numbers (Table 1 according to it), result is given in the table 4 below:

Table 4. Fuzzy numbers of the importance weight of the criteria

\begin{tabular}{lccc}
\hline & \multicolumn{3}{c}{ Experts } \\
\hline & $e_{11}$ & $e_{12}$ & $e_{13}$ \\
$C_{11}^{\prime}$ & $(0.9,1,1)$ & $(0.9,1,1)$ & $(0.7,0.9,1)$ \\
$C_{12}^{\prime}$ & $(0.5,0.7,0.9)$ & $(0.5,0.7,0.9)$ & $(0.7,0.9,1)$ \\
$C_{13}^{\prime}$ & $(0.3,0.5,0.7)$ & $(0.3,0.5,0.7)$ & $(0.1,0.3,0.5)$ \\
$C_{14}^{\prime}$ & $(0.5,0.7,0.9)$ & $(0.7,0.9,1)$ & $(0.5,0.7,0.9)$ \\
$C_{15}^{\prime}$ & $(0.3,0.5,0.7)$ & $(0.7,0.9,1)$ & $(0.1,0.3,0.5)$ \\
\hline
\end{tabular}


Basheleishvili et al. / The Development of a Model for Decision Support System of Assessment and Selection of University Academic Staff

Table 5. Fuzzy weight for all criteria

\begin{tabular}{lccccc}
\hline & $W_{11}^{\prime}$ & $W_{12}^{\prime}$ & $W_{13}^{\prime}$ & $W_{14}^{\prime}$ & $W_{15}^{\prime}$ \\
\hline weight & $(0.83,0.97,1)$ & $(0.57,0.77,0.93)$ & $(0.23,0.43,0.63)$ & $(0.57,0.77,0.93)$ & $(0.37,0.57,0.73)$ \\
\hline
\end{tabular}

The experts use the linguistic weighting variables (See Table 2) to determine the priority of each criterion and the alternative is summarized in Table 6.

\begin{tabular}{|c|c|c|c|c|}
\hline \multirow{6}{*}{$p_{11}^{\prime}$} & & $e_{11}$ & $e_{12}$ & $e_{13}$ \\
\hline & $C_{11}^{\prime}$ & MP & MG & $\mathrm{MG}$ \\
\hline & $C_{12}^{\prime}$ & MP & $\mathrm{F}$ & $\mathrm{F}$ \\
\hline & $C_{13}^{\prime}$ & $\mathrm{G}$ & $\mathrm{G}$ & $\mathrm{MG}$ \\
\hline & $C_{14}^{\prime}$ & $\mathrm{F}$ & MP & $\mathrm{F}$ \\
\hline & $C_{15}^{\prime}$ & $\mathrm{G}$ & $\mathrm{G}$ & MG \\
\hline \multirow{6}{*}{$p_{12}^{\prime}$} & & $e_{11}$ & $e_{12}$ & $e_{13}$ \\
\hline & $C_{11}^{\prime}$ & $\mathrm{F}$ & MG & $\mathrm{MG}$ \\
\hline & $C_{12}^{\prime}$ & MP & MP & MP \\
\hline & $C_{13}^{\prime}$ & $\mathrm{G}$ & MG & $\mathrm{G}$ \\
\hline & $C_{14}^{\prime}$ & $\mathrm{G}$ & $\mathrm{G}$ & $\mathrm{MG}$ \\
\hline & $C_{15}^{\prime}$ & $\mathrm{F}$ & $\mathrm{F}$ & $\mathrm{F}$ \\
\hline \multirow{6}{*}{$p_{13}^{\prime}$} & & $e_{11}$ & $e_{12}$ & $e_{13}$ \\
\hline & $C_{11}^{\prime}$ & $\mathrm{MG}$ & $\mathrm{F}$ & $\mathrm{MG}$ \\
\hline & $C_{12}^{\prime}$ & $\mathrm{F}$ & MP & $\mathrm{MG}$ \\
\hline & $C_{13}^{\prime}$ & $\mathrm{G}$ & MG & $\mathrm{G}$ \\
\hline & $C_{14}^{\prime}$ & $\mathrm{MG}$ & $\mathrm{F}$ & $\mathrm{MG}$ \\
\hline & $C_{15}^{\prime}$ & $\mathrm{G}$ & MG & $\mathrm{G}$ \\
\hline \multirow{6}{*}{$p_{14}^{\prime}$} & & $e_{11}$ & $e_{12}$ & $e_{13}$ \\
\hline & $C_{11}^{\prime}$ & $\mathrm{P}$ & $\mathrm{F}$ & $\mathrm{MG}$ \\
\hline & $C_{12}^{\prime}$ & $\mathrm{F}$ & MP & $\mathrm{MG}$ \\
\hline & $C_{13}^{\prime}$ & $\mathrm{G}$ & $\mathrm{MG}$ & $\mathrm{G}$ \\
\hline & $C_{14}^{\prime}$ & MG & $\mathrm{F}$ & $\mathrm{MG}$ \\
\hline & $C_{15}^{\prime}$ & $\mathrm{G}$ & MG & VG \\
\hline \multirow{6}{*}{$p_{15}^{\prime}$} & & $e_{11}$ & $e_{12}$ & $e_{13}$ \\
\hline & $C_{11}^{\prime}$ & MP & $\mathrm{P}$ & MP \\
\hline & $C_{12}^{\prime}$ & $\mathrm{F}$ & MP & $\mathrm{MG}$ \\
\hline & $C_{13}^{\prime}$ & $\mathrm{G}$ & MG & $\mathrm{G}$ \\
\hline & $C_{14}^{\prime}$ & $\mathrm{F}$ & $\mathrm{F}$ & $\mathrm{MG}$ \\
\hline & $C_{15}^{\prime}$ & $\mathrm{G}$ & $\mathrm{MG}$ & VG \\
\hline
\end{tabular}

Changing the linguistic evaluation (shown in Table 2) to the triangular fuzzy numbers (Table 7) and then build a fuzzy decision matrix (Table 8):

Table 7

\begin{tabular}{|c|c|c|c|c|}
\hline & & $\mathrm{e}_{11}$ & $\mathrm{e}_{12}$ & $\mathrm{e}_{13}$ \\
\hline \multirow{5}{*}{$p_{11}^{\prime}$} & $C_{11}^{\prime}$ & $(1,3,5)$ & $(5,7,9)$ & $(5,7,9)$ \\
\hline & $C_{12}^{\prime}$ & $(1,3,5)$ & $(3,5,7)$ & $(3,5,7)$ \\
\hline & $C_{13}^{\prime}$ & $(7,9,10)$ & $(7,9,10)$ & $(5,7,9)$ \\
\hline & $C_{14}^{\prime}$ & $(3,5,7)$ & $(1,3,5)$ & $(3,5,7)$ \\
\hline & $C_{15}^{\prime}$ & $(7,9,10)$ & $(7,9,10)$ & $(5,7,9)$ \\
\hline \multirow{5}{*}{$p_{12}$} & $C_{11}^{\prime}$ & $(3,5,7)$ & $(5,7,9)$ & $(5,7,9)$ \\
\hline & $C_{12}^{\prime}$ & $(1,3,5)$ & $(1,3,5)$ & $(1,3,5)$ \\
\hline & $C_{13}^{\prime}$ & $(7,9,10)$ & $(5,7,9)$ & $(7,9,10)$ \\
\hline & $C_{14}^{\prime}$ & $(7,9,10)$ & $(7,9,10)$ & $(5,7,9)$ \\
\hline & $C_{15}^{\prime}$ & $(3,5,7)$ & $(3,5,7$ & $(3,5,7$ \\
\hline \multirow{5}{*}{$p_{13}^{\prime}$} & $C_{11}^{\prime}$ & $(5,7,9)$ & $(3,5,7)$ & $(5,7,9)$ \\
\hline & $C_{12}^{\prime}$ & $(3,5,7)$ & $(1,3,5)$ & $(5,7,9)$ \\
\hline & $C_{13}^{\prime}$ & $(7,9,10)$ & $(5,7,9)$ & $(7,9,10)$ \\
\hline & $C_{14}^{\prime}$ & $(5,7,9)$ & $(3,5,7)$ & $(5,7,9)$ \\
\hline & $C_{15}^{\prime}$ & $(7,9,10)$ & $(5,7,9)$ & $(7,9,10)$ \\
\hline \multirow{5}{*}{$p_{14}^{\prime}$} & $C_{11}^{\prime}$ & $(0,1,3)$ & $(3,5,7)$ & $(5,7,9)$ \\
\hline & $C_{12}^{\prime}$ & $(3,5,7)$ & $(1,3,5)$ & $(5,7,9)$ \\
\hline & $C_{13}^{\prime}$ & $(7,9,10)$ & $(5,7,9)$ & $(7,9,10)$ \\
\hline & $C_{14}^{\prime}$ & $(5,7,9)$ & $(3,5,7)$ & $(5,7,9)$ \\
\hline & $C_{15}^{\prime}$ & $(7,9,10)$ & $(5,7,9)$ & $(9,10,0)$ \\
\hline \multirow{5}{*}{$p_{15}^{\prime}$} & $C_{11}^{\prime}$ & $(1,3,5)$ & $(0,1,3)$ & $(1,3,5)$ \\
\hline & $C_{12}^{\prime}$ & $(3,5,7)$ & $(1,3,5)$ & $(5,7,9)$ \\
\hline & $C_{13}^{\prime}$ & $(7,9,10)$ & $(5,7,9)$ & $(7,9,10)$ \\
\hline & $C_{14}^{\prime}$ & $(3,5,7)$ & $(3,5,7)$ & $(5,7,9)$ \\
\hline & $C_{15}^{\prime}$ & $(7,9,10)$ & $(5,7,9)$ & $(9,10,0)$ \\
\hline
\end{tabular}


Basheleishvili et al. / The Development of a Model for Decision Support System of Assessment and Selection of University Academic Staff

Table 8. Fuzzy decision matrix

\begin{tabular}{lccccc}
\hline & $C_{11}^{\prime}$ & $C_{12}^{\prime}$ & $C_{13}^{\prime}$ & $C_{14}^{\prime}$ & $C_{15}^{\prime}$ \\
\hline$p_{11}^{\prime}$ & $(3.67,5.67,7.67)$ & $(2.33,4.33,6.33)$ & $(6.33,8.33,9.67$ & $(2.33,4.33,6.33)$ & $(6.33,8.33,9.67)$ \\
$p_{12}^{\prime}$ & $(4.33,6.33,8.33)$ & $(1,3,5)$ & $(6.33,8.33,9.67)$ & $(6.33,8.33,9.67)$ & $(3,5,7)$ \\
$p_{13}^{\prime}$ & $(4.33,6.33,8.33)$ & $(3,5,7)$ & $(6.33,8.33,9.67)$ & $(4.33,6.33,8.33)$ & $(6.33,8.33,9.67)$ \\
$p_{14}^{\prime}$ & $(2.67,4.33,6.33)$ & $(3,5,7)$ & $(6.33,8.33,9.67)$ & $(4.33,6.33,8.33)$ & $(4.33,6.33,8.33)$ \\
$p_{15}^{\prime}$ & $(0.67,2.33,4.33)$ & $(3,5,7)$ & $(6.33,8.33,9.67)$ & $(3.67,5.67,7.67)$ & $(7,8.67,6.33)$ \\
\hline
\end{tabular}

Construct a normalized fuzzy decision matrix (Table 9):

Table 9. Normalized fuzzy decision matrix

\begin{tabular}{lccccc}
\hline & $C_{11}^{\prime}$ & $C_{12}^{\prime}$ & $C_{13}^{\prime}$ & $C_{14}^{\prime}$ & $C_{15}^{\prime}$ \\
\hline$p_{11}^{\prime}$ & $(0.37,0.657,0.92)$ & $(0.19,0.47,0.84)$ & $(0.15,0.37,0.63)$ & $(0.14,0.34,0.61)$ & $(0.24,0.49,0.73)$ \\
$p_{12}^{\prime}$ & $(0.43,0.73,1)$ & $(0.08,0.33,0.66)$ & $(0.15,0.37,0.63)$ & $(0.37,0.66,0.93)$ & $(0.11,0.29,0.53)$ \\
$p_{13}^{\prime}$ & $(0.43,0.73,1)$ & $(0.24,0.55,0.93)$ & $(0.15,0.37,0.63)$ & $(0.25,0.50,0.80)$ & $(0.24,0.49,0.73)$ \\
$p_{14}^{\prime}$ & $(0.35,0.50,0.76)$ & $(0.24,0.55,0.93)$ & $(0.15,0.37,0.63)$ & $(0.25,0.50,1)$ & $(0.16,0.37,0.63)$ \\
$p_{15}^{\prime}$ & $(0.07,0.27,0.52)$ & $(0.24,0.55,0.93)$ & $(0.15,0.37,0.63)$ & $(0.21,0.45,0.74)$ & $(0.27,0.51,0.48)$ \\
\hline
\end{tabular}

Construct a weighted normalized fuzzy decision matrix (Table 10):

Table 10. Weighted normalized fuzzy decision matrix

\begin{tabular}{cccccc}
\hline & $C_{11}^{\prime}$ & $C_{12}^{\prime}$ & $C_{13}^{\prime}$ & $C_{14}^{\prime}$ & $C_{15}^{\prime}$ \\
\hline$p_{11}^{\prime}$ & $(0.37,0.657,0.92)$ & $(0.19,0.47,0.84)$ & $(0.15,0.37,0.63)$ & $(0.14,0.34,0.61)$ & $(0.24,0.49,0.73)$ \\
$p_{12}^{\prime}$ & $(0.43,0.73,1)$ & $(0.08,0.33,0.66)$ & $(0.15,0.37,0.63)$ & $(0.37,0.66,0.93)$ & $(0.11,0.29,0.53)$ \\
$p_{13}^{\prime}$ & $(0.43,0.73,1)$ & $(0.24,0.55,0.93)$ & $(0.15,0.37,0.63)$ & $(0.25,0.50,0.80)$ & $(0.24,0.49,0.73)$ \\
$p_{14}^{\prime}$ & $(0.35,0.50,0.76)$ & $(0.24,0.55,0.93)$ & $(0.15,0.37,0.63)$ & $(0.25,0.50,1)$ & $(0.16,0.37,0.63)$ \\
$p_{15}^{\prime}$ & $(0.07,0.27,0.52)$ & $(0.24,0.55,0.93)$ & $(0.15,0.37,0.63)$ & $(0.21,0.45,0.74)$ & $(0.27,0.51,0.48)$ \\
\hline
\end{tabular}

The next step is to get the fuzzy positive ideal solutions (FPIS), $\left(\mathrm{A}^{+}\right)$and fuzzy negative ideal solutions (FNIS), $\left(\mathrm{A}^{-}\right) . \mathrm{A}^{+}=(1,1,1,1,1)$ and $\mathrm{A}^{-}=$ $(0,0,0,0,0)$. Calculate the distance of the alternatives from $\left(\mathrm{A}^{+}\right)$and $\left(\mathrm{A}^{-}\right)$. The next step calculated the correlation coefficients for each alternative (Table 11).

\begin{tabular}{lcccc}
\hline & $\mathrm{d}^{+}$ & $\mathrm{d}^{-}$ & $\mathrm{CC}_{1 \mathrm{k}}$ & $\mathrm{Rank}$ \\
\hline$p_{11}^{\prime}$ & 2.843588 & 2.6281 & 0.4803 & 4 \\
$p_{12}^{\prime}$ & 2.812948 & 2.6734 & 0.4873 & 3 \\
$p_{13}^{\prime}$ & 2.596798 & 2.9254 & 0.5297 & 1 \\
$p_{14}^{\prime}$ & 2.804767 & 2.7396 & 0.4941 & 2 \\
$p_{15}^{\prime}$ & 3.058351 & 2.3599 & 0.4355 & 5 \\
\hline
\end{tabular}

Let's select three of the highest $\mathrm{CC}_{1 \mathrm{k}}$ value $-p_{13}^{\prime}, p_{14}^{\prime}$ ,$p_{12}^{\prime}$.

\section{Conclusions}

The paper describes the assessment and selection model of the university's academic staff, which allows us for making the best choice from the applicants on the basis of the expert assessments. Based on this model, we can establish the decision-making support system for the assessment and selection of academic staff. The shortcoming of this model is that it is based entirely on the expert assessments and depends on the expert's qualification and his/her objectivity. I plan to carry out research to eliminate these shortcomings. 
Basheleishvili et al. / The Development of a Model for Decision Support System of Assessment and Selection of University Academic Staff

\section{References}

Basheleishvili, I. (2018). Development of Method of Multifunctional Personnel Assessment Using a Topsis Method. Journal of Technical Science and Technologies, $6(2)$.

Basheleishvili, I. (2018). The Algorithm of Selection and Functions Distribution of Multifunctional Personnel-Case when the Number of Functions is Greater than the Number of Personnel. Journal of Technical Science and Technologies, 6(2).

Basheleishvili, I., \& Bardavelidze, A. (2018). Development of Human Resource Assessment and Selection Model for Computer System Design. International Journal of Computer (IJC), 30(1), 50-58.

Ehrgott, M., Figueira, J., \& Greco, S. (2010). Trends in multiple criteria decision analysis (Vol. 6, pp. 10-34). New York: Springer.

Junior, F. R. L., Osiro, L., \& Carpinetti, L. C. R. (2014). A comparison between Fuzzy AHP and Fuzzy TOPSIS methods to supplier selection. Applied Soft Computing, 21, 194-209.

Keenan, P., McGarraghy, S., McNamara, C., Phelan, M., \& Schools, U. B. (2004). Human resource management DSS. In International Conference DSS2004 (pp. 525-534).

Madi, E. N., Tap, A. O. M., \& Osman, A. (2011, July). Fuzzy TOPSIS method in the selection of investment boards by incorporating operational risks. In Proceedings of the world congress on engineering (Vol. 1, No. 1, pp. 291295).

Meinert, D. B., \& Davis, D. L. (1989). Human Resource Decision Support Systems (HRDSS): Integrating Decision Support and Human Resource Information Systems. Information Resources Management Journal (IRMJ), 2(1), 41-48.

Moses, I. (1988). Academic Staff Evaluation and Development. A University Case Study. University of Queensland Press, 250 Commercial Street, Manchester, NH 03101.

Sun, C. C., \& Lin, G. T. (2009). Using fuzzy TOPSIS method for evaluating the competitive advantages of shopping websites. Expert Systems with Applications, 36(9), 1176411771.

Zhang, X., Ma, W., \& Chen, L. (2014). New similarity of triangular fuzzy number and its application. The Scientific World Journal, 2014. 